\title{
Identification of human intestinal parasites affecting an asymptomatic peri-urban Argentinian population using multi-parallel quantitative real-time polymerase chain reaction
}

Rubén O. Cimino ${ }^{1,2 \dagger}$, Rebecca Jeun ${ }^{3 \dagger}$, Marisa Juarez ${ }^{1,4}$, Pamela S. Cajal ${ }^{1}$, Paola Vargas ${ }^{1}$, Adriana Echazú1, Patricia E. Bryan ${ }^{3}$, Julio Nasser ${ }^{1,2}$, Alejandro Krolewiecki ${ }^{1}$ and Rojelio Mejia $2,3^{*}$

\begin{abstract}
Background: In resource-limited countries, stool microscopy is the diagnostic test of choice for intestinal parasites (soil-transmitted helminths and/or intestinal protozoa). However, sensitivity and specificity is low. Improved diagnosis of intestinal parasites is especially important for accurate measurements of prevalence and intensity of infections in endemic areas.

Methods: The study was carried out in Orán, Argentina. A total of 99 stool samples from a local surveillance campaign were analyzed by concentration microscopy and McMaster egg counting technique compared to the analysis by multi-parallel quantitative real-time polymerase chain reaction (qPCR). This study compared the performance of qPCR assay and stool microscopy for 8 common intestinal parasites that infect humans including the helminths Ascaris lumbricoides, Ancylostoma duodenale, Necator americanus, Strongyloides stercoralis, Trichuris trichiura, and the protozoa Giardia lamblia, Cryptosporidium parvum/hominis, and Entamoeba histolytica, and investigated the prevalence of polyparasitism in an endemic area.

Results: qPCR showed higher detection rates for all parasites as compared to stool microscopy except T. trichiura. Species-specific primers and probes were able to distinguish between A. duodenale (19.1\%) and N. americanus (36.4\%) infections. There were $48.6 \%$ of subjects co-infected with both hookworms, and a significant increase in hookworm DNA for A. duodenale versus $N$. americanus (119.6 fg/ $\mu \mathrm{L}: 0.63 \mathrm{fg} / \mu \mathrm{L}, P<0.001)$ respectively. qPCR outperformed microscopy by the largest margin in G. lamblia infections (63.6 \% versus $8.1 \%, P<0.05$ ). Polyparasitism was detected more often by qPCR compared to microscopy (64.7\% versus $24.2 \%, P<0.05$ ).

Conclusions: Multi-parallel GPCR is a quantitative molecular diagnostic method for common intestinal parasites in an endemic area that has improved diagnostic accuracy compared to stool microscopy. This first time use of multi-parallel gPCR in Argentina has demonstrated the high prevalence of intestinal parasites in a peri-urban area. These results will contribute to more accurate epidemiological survey, refined treatment strategies on a public scale, and better health outcomes in endemic settings.
\end{abstract}

Keywords: Intestinal parasites, Molecular diagnostics, Prevalence, Argentina, Hookworm speciation, Real-time PCR

\footnotetext{
* Correspondence: rmejia@bcm.edu

${ }^{\dagger}$ Equal contributors

${ }^{2}$ Cátedra de Química Biológica, Facultad de Ciencias Naturales, Universidad

Nacional de Salta, Oran, Argentina

${ }^{3}$ National School of Tropical Medicine, Baylor College of Medicine, Houston,

TX, USA

Full list of author information is available at the end of the article
}

\section{Biomed Central}

(c) 2015 Cimino et al. This is an Open Access article distributed under the terms of the Creative Commons Attribution License (http://creativecommons.org/licenses/by/4.0), which permits unrestricted use, distribution, and reproduction in any medium, provided the original work is properly credited. The Creative Commons Public Domain Dedication waiver (http:// creativecommons.org/publicdomain/zero/1.0/) applies to the data made available in this article, unless otherwise stated. 


\section{Background}

The World Health Organization (WHO) classifies most parasitic diseases as Neglected Tropical Diseases (NTD) [1]. Soil-transmitted helminths $(\mathrm{STH})$ and intestinal protozoa are distributed widely throughout the world and are more prevalent in tropical and sub-tropical regions. STH (Ascaris lumbricoides, Ancylostoma duodenale, Necator americanus, Strongyloides stercoralis, and Trichuris trichiura) affect more than 2 billion people worldwide [2]. These species produce a wide array of symptoms, from asymptomatic (not reporting intestinal complaints) to including diarrhea, abdominal pain, general malaise, and weakness that may impact learning capacities and impaired physical growth [3-5]. Hookworms cause chronic intestinal blood loss that results in anemia, significantly impacting health [6]. Infections with pathogenic intestinal protozoa infections, primarily Giardia lamblia and Entamoeba histolytica, are also of considerable public health importance. In northern Argentina, mainly in the Yungas rainforest and Chaco regions, recent reports indicate prevalence rates of over $20 \%$, with some areas approaching $50 \%[7,8]$.

The standard diagnostic method for gastrointestinal parasites is direct microscopic examination of stool samples. For STH infections, the recommended technique for microscopic diagnosis is Kato-Katz, except for $S$. stercoralis, for which the Baermann, agar plate, and Harada Mori methods are recommended [9]. Other fecal egg count (FEC) techniques such as McMaster and the recently developed FLOTAC and Mini-FLOTAC have also been successfully used for diagnosis of STH infections in humans $[10,11]$.

Recently, molecular tools such as quantitative realtime PCR (qPCR) have improved diagnosis of gastrointestinal parasites $[12,13]$. Multi-parallel and multiplex qPCR have the advantage of enabling detection of multiple parasite species using a single stool sample as well as the ability to determine the intensity of infection of each species $[12,13]$. This study, the first use of multiparallel qPCR in Argentina, sought to compare the performance of multi-parallel qPCR assay and stool microscopy in an endemic area for 8 common intestinal parasites including the intestinal helminths Ascaris lumbricoides, Ancylostoma duodenale, Necator americanus, Trichuris trichiura, Strongyloides stercoralis and the protozoa Cryptosporidium parvum/hominis, Entamoeba histolytica, and Giardia lamblia. Of particular interest is the diversity of intestinal parasite infections within the study site, Orán, Argentina, as it is a peri-urban locale with higher population density than a rural area, but fewer community services such as sanitation and paved roads than an urban center. Although qPCR has previously been compared to stool microscopy in Ecuador [12], it was important to field test qPCR at this new site in Argentina. Especially since McMasters method is the diagnostic standard for microscopy in Argentina and has never been previously compared to qPCR.

This study also investigated the prevalence of polyparasitism and the differences in the epidemiology of hookworm infection. Hookworm infection is of special interest from a diagnostic perspective because infection can result in anemia and childhood stunting [2-6]. Stool microscopy cannot distinguish between Necator americanus and Ancylostoma duodenale ova, while qPCR can differentiate species and co-infections without waiting for larvae development [12].

\section{Methods}

\section{Ethics, consent and permissions}

This study was approved by the internal review boards of Baylor College of Medicine and Universidad Nacional de Salta Argentina.

Informed written consent was obtained from each participant or from a parent/guardian. Anti-parasitic treatment, based on microscopy findings, was provided per standard of care in the region. The majority of subjects were of preschool age and did not receive standard mass drug administration of anthelmintics. Study design and protocols were approved by the bioethics committee of Universidad Nacional de Salta, Argentina.

\section{Sample collection}

Fecal samples were collected from a random cohort of 99 asymptomatic subjects ages 7 months through 57 years old (geometric mean, 7.2 years old) living in the Orán department, Salta Province, Argentina. Samples were collected from September through December 2012. Stool was then frozen without fixatives at $-80{ }^{\circ} \mathrm{C}$ until used for DNA extraction and retrospective study with qPCR.

\section{Microscopy}

Stool samples were examined by Telemann concentration, as previously described, and McMaster methods at the time of stool collection [14]. The McMaster method was performed following the standard procedure: $2 \mathrm{~g}$ of feces were filtered and homogenized with $30 \mathrm{ml}$ of saturated saline. Two flotation chambers (1 ml each) were filled for each sample. Eggs were allowed to float for 3 min then counted. This number was multiplied by 50 for the egg per gram count (epg). qPCR was compared to any positive microscopy sample using the concentration and McMaster method.

\section{DNA extraction}

DNA was extracted from $50 \mathrm{mg}$ stool by using the FastPrep ${ }^{\oplus}$ Spin Kit for Soil (MP Biomedicals, Santa Ana, 
CA) according to manufacturer's instructions for all parasites except $T$. trichiura. An additional step required for the extraction of T. trichiura DNA was performed as previously described [12]. All samples were processed in Salta, Argentina.

\section{Multi-parallel qPCR}

Multi-parallel qPCR was performed as previously described [12] with the same species-specific primers and FAM-labeled minor groove binder probes (Applied Biosystems, Foster City, CA). Samples were run on an ABI ViiA7 PCR in Houston, Texas with default parameters and 40 cycles. DNA concentrations were calculated using a standard curve.

\section{Statistical analysis}

All statistical analyses were performed with Prism version 6.0f (GraphPad, La Jolla, CA). Comparisons of microscopy with qPCR were analyzed by using Fisher's exact test, and $P$ values $<0.05$ were considered statistically significant. Correlations were estimated by calculation of Spearman's rank correlation coefficients. Welch's unpaired t-test was used in comparisons of DNA concentrations.

\section{Results}

\section{Comparison of qPCR with microcopy}

The results of $\mathrm{qPCR}$ and microscopy for all studied intestinal parasites are summarized in Table 1. qPCR was significantly more sensitive than stool microscopy in identifying $S$. stercoralis $(21.2 \%$ versus $3.0 \%, P<0.05)$, G. lamblia (63.6 \% versus $8.1 \%, P<0.05)$, and total hookworm infections (37.4 \% versus $21.2 \%, P<0.05)$. A stark difference was noted with G. lamblia as qPCR detected more positive samples than stool microscopy by a factor of 8 with a sensitivity of 87.5 and $97.2 \%$ negative predictive value (NPV). Both methods identified similar number of positive samples of A. lumbricoides, but qPCR detected three more than concentration microscopy and nine more than McMaster method. For A. lumbricoides, qPCR had a $91.3 \%$ sensitivity and $90.5 \%$ NPV. For S. stercoralis, qPCR had a $100 \%$ sensitivity and NPV.

Additionally, qPCR has the ability to distinguish $A$. duodenale and $N$. americanus. N. americanus (36.4 \%) was more prevalent in this study population than $A$. duodenale (19.1\%). Significantly more infections with combined hookworm species were detected with qPCR compared to concentration and McMaster method (37.4 \% versus $21.2 \%, P<0.05)$, respectively and had a $95.5 \%$ sensitivity and $98.4 \%$ NPV.

The only intestinal parasite that stool microscopy detected more frequently, was $T$. trichiura though the difference was not significant $(P=0.118)$. No Cryptosporidium parvum/hominis positive samples were identified in the study population by qPCR. Only one E. histolytica positive sample was detected by qPCR though it was not seen by microscopy. DNA concentrations were higher in those samples also found to be positive by microscopy except in the case of $A$. duodenale, but did not reach statistical significance.

\section{Hookworm studies}

Since qPCR has the ability to distinguish between hookworm species, $A$. duodenale and $N$. americanus, this study assessed the DNA concentration found in positive samples of both species (Fig. 1). The DNA concentration was significantly higher with $A$. duodenale as compared to $N$. americanus (geometric mean of $A$. duodenale: $119.6 \mathrm{fg} / \mu \mathrm{L}$ versus $N$. americanus: $0.63 \mathrm{fg} / \mu \mathrm{L}, P<0.001$ ). There were 18/37 (48.6\%) samples containing both $A$. duodenale and $N$. americanus DNA, without any

Table 1 Comparison of intestinal parasite detection by stool qPCR and microscopy in 99 subjects

\begin{tabular}{|c|c|c|c|c|c|}
\hline Parasite & $\begin{array}{l}\text { No. positive by } \\
\text { multi-parallel qPCR (\%) }\end{array}$ & $\begin{array}{l}\text { No. positive by } \\
\text { concentration } \\
\text { microscopy (\%) }\end{array}$ & $\begin{array}{l}\text { No. positive by } \\
\text { McMaster } \\
\text { microscopy (\%) }\end{array}$ & $\begin{array}{l}\text { DNA concentrations }(\mathrm{fg} / \mu \mathrm{L}) \text { in } \\
\text { stool positive by microscopy, } \\
\text { median (range) }\end{array}$ & $\begin{array}{l}\text { DNA concentrations ( } \mathrm{fg} / \mu \mathrm{L}) \text { in } \\
\text { stool negative by microscopy, } \\
\text { median (range) }\end{array}$ \\
\hline Ascaris lumbricoides & $56 / 99(56.5)$ & $53 / 99(53.5)$ & 47/99 (47.5) & $1.02(0.001-42.3)$ & $0.48(0.003-10.431)$ \\
\hline $\begin{array}{l}\text { Cryptosporidium } \\
\text { parvum/hominis }\end{array}$ & 0/99 (0) & NA & NA & NA & NA \\
\hline Ancylostoma duodenale & 19/99 (19.1) & NA & NA & $55.1(5.87-278.2)^{a}$ & $106.9(26.2-384.2)$ \\
\hline Necator americanus & 36/99 (36.4) & NA & NA & $12.5(0.024-278.2)^{a}$ & $1.59(0.001-239.5)$ \\
\hline Hookworm* & $37 / 99(37.4)$ & $21 / 99(21.2)^{a}$ & $21 / 99(21.2)^{a}$ & NA & NA \\
\hline Strongyloides stercoralis* & $21 / 99(21.2)$ & 3/99 (3.0) & NA & $65.9(14.7-123.6)$ & $0.22(0.0009-48.6)$ \\
\hline Giardia lamblia* & 63/99 (63.6) & 8/99 (8.1) & NA & $5.27(0.02-1847.4)$ & $0.27(0.007-5697.8)$ \\
\hline Entamoeba histolytica & 1/99 (1.0) & 0/99 (0) & NA & 0.003 & 0 \\
\hline Trichuris trichiura & 1/99 (1.0) & 4/99 (4.0) & 6/99 (6.1) & 0.001 & 0 \\
\hline
\end{tabular}

NA Not applicable

*Indicates statistically significant difference $(P<0.05)$

${ }^{a}$ Microscopy is unable to distinguish between the two hookworm species, A. duodenale and N. americanus 


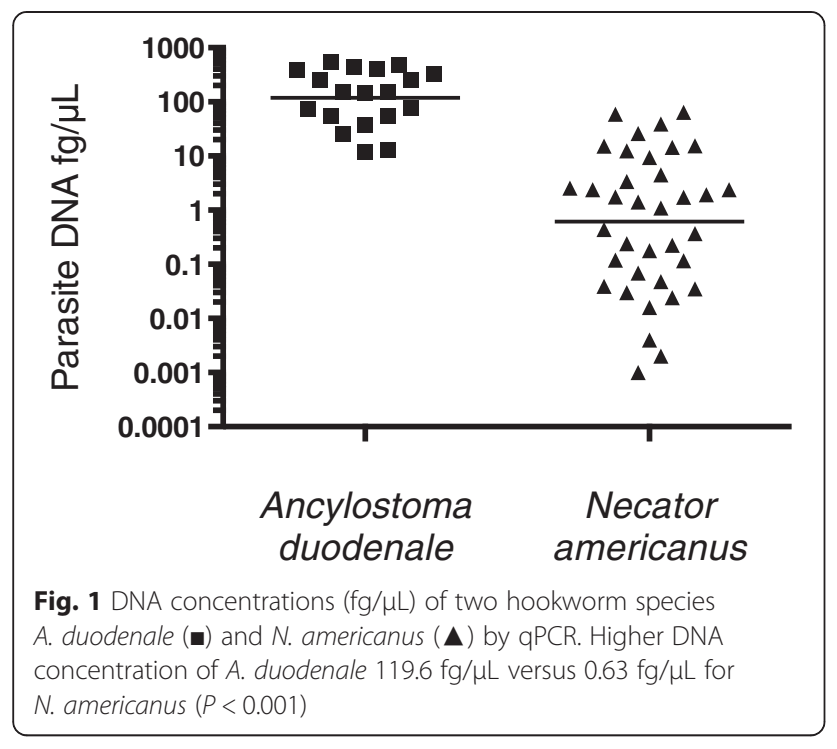

correlation between the two species' DNA concentrations $(P>0.05)$.

Comparison of hookworm egg counts by the McMaster method with hookworm DNA concentration (geometric mean) in samples positive by both methods showed a statistically significant correlation (Fig. 2, $r=$ $0.632, P=0.0028)$. The average DNA concentration of $A$. duodenale and $N$. americanus in co-infected individuals was used in this correlation (Fig. 2).

\section{Detecting polyparasitism by qPCR}

The vast majority of the study population had positive stool samples for at least one kind of intestinal parasite infection by qPCR and/or microscopy (96/99, $97.0 \%)$. Microscopy was only able to detect two or more intestinal parasite species in $24 / 99(24.2 \%)$ samples as

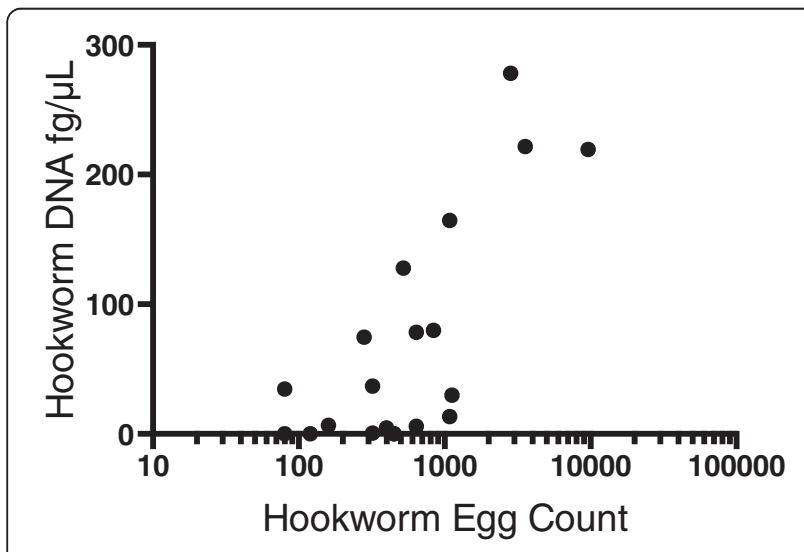

Fig. 2 Correlation of hookworm DNA concentration $(f g / \mu L)$ with hookworm egg count by McMaster method (eggs per gram) $(r=0.632, P=0.0028)$. Only samples positive by $q P C R$ are shown compared to 64/99 (64.7\%) samples identified by qPCR (Fig. 3a). Microscopy distinguished a maximum of three different intestinal parasites in the same sample (2/99, $2.0 \%)$ compared to $15 / 99$ (15.2 \%) for qPCR. While qPCR was even able to detect up to four different species in the same samples $(10 / 99,10.1 \%)$, microscopy did not detect any. All polyparasitic comparisons were statistically significant $(P<0.05)$.

In terms of specific co-infections, qPCR identified more cases of co-infection by G. lamblia/A. lumbricoides (32 versus $3, P<0.05)$, G. lamblia/hookworm (28 versus $2, P<0.05$ ), and $A$. lumbricoides/hookworm (4 versus $1, P>0.05$ ) than microscopy, respectively (Fig. $3 \mathrm{~b}$ ). G. lamblia/A. lumbricoides was the most commonly encountered co-infection by both qPCR and microscopy though qPCR detected more samples by a factor of 10 . G. lamblia and A. lumbricoides were also the most prevalent species in the study population by both microscopy and qPCR. The same four parasites (G. lamblia, S. stercoralis, $N$. americanus, and A. duodenale) were found in all ten samples that were positive by qPCR for a quadruple infection.

\section{Discussion}

The geographic distribution of intestinal parasite infections overlaps significantly with resource-poor nations that lack consistent access to clean water, sanitation, and health care [15]. Reducing morbidity associated with intestinal parasites requires reliable data on prevalence. This is difficult to accomplish when the diagnostic standard, stool microscopy, often lacks sensitivity and specificity. Multi-parallel qPCR was developed to improve upon the diagnostic sensitivity of microscopy, especially in epidemiological studies, and to complement microscopy as a diagnostic tool in resource-limited settings. This study compared qPCR assay to microscopy in the diagnosis of 8 common intestinal parasites. Particularly, the degree of hookworm infection and the prevalence of polyparasitism were investigated.

Extremely high rates of intestinal parasite infections were detected, both by microscopy and qPCR. Although, qPCR detected a higher number of total infections, the high rates of infection were especially noteworthy in an asymptomatic population. qPCR detected significantly more hookworm, S. stercoralis, and G. lamblia infections than microscopy. qPCR and microscopy performed similarly for A. lumbricoides, likely because the eggs are more easily visualized than other species. For STH, the prevalence of ascariasis and hookworm infection by qPCR exceeds the estimated national prevalence of 5-24.9\% [16]. Consideration should also be given to geographical region, since temperature, area, and local populations have varied parasitosis prevalence [17]. There were none or low rates of C. parvum/hominis, E. 


\section{A Microscopy}
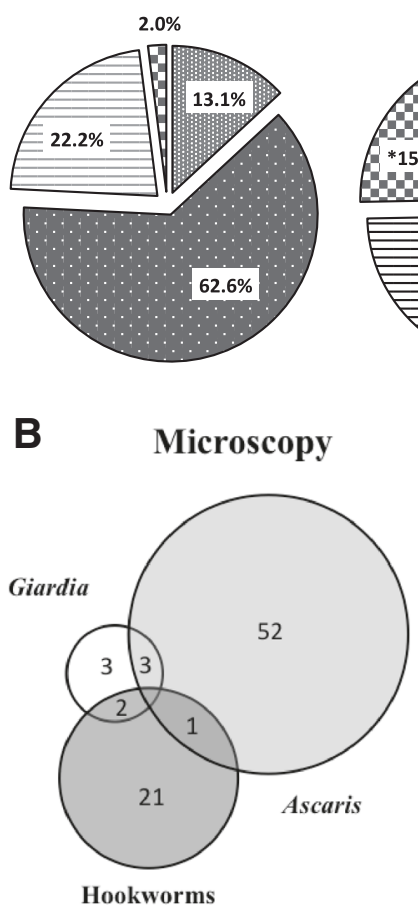

\section{qPCR}

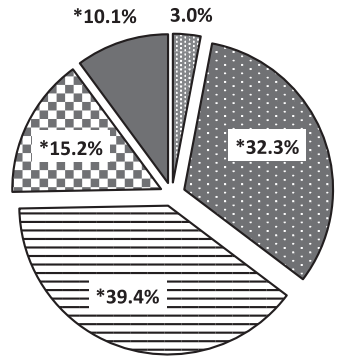

圈0 parasites

1 parasite

目 2 parasites

曰3 parasites

$\square 4$ parasites

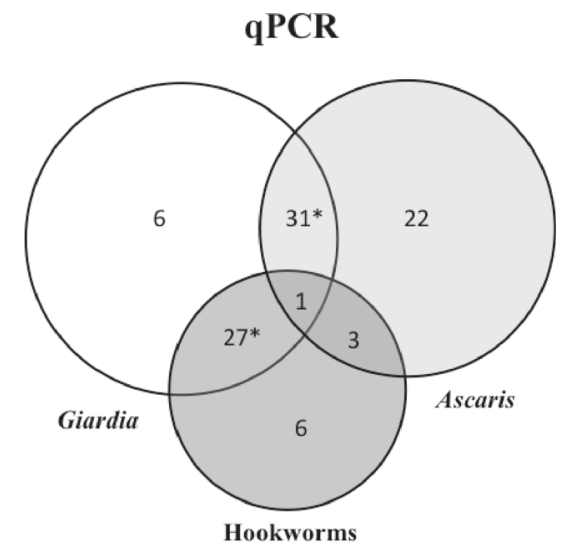

Fig. 3 Detection of polyparasitism by quantitative multi-parallel real-time polymerase chain reaction (qPCR) and microscopy in 99 patients from Argentina. a Percentages of stools positive for 0-4 parasites by microscopy or qPCR. *Indicates statistically significant difference $(P<0.05)$. b Increased detection of the three most prevalent intestinal parasite infections (in a Venn diagram) by microscopy and qPCR

histolytica, and T. trichiura identified by both $\mathrm{qPCR}$ and microscopy. T. trichiura was the only species for which microscopy detected more positive samples which may suggest loss of yield in the extra step of processing required in the qPCR protocol. Since qPCR has previously been shown to be more sensitive than microscopy in detecting T. trichiura, lack of primer specificity is likely not the issue [12]. Another possibility for this discordance is subtle genetic differences in the ITS-1 target region of Trichuris trichiura found in Argentina. Primer/probe combinations were field tested and optimized for use in Ecuador [12]. This may explain the lack of qPCR sensitivity and further research is needed.

A benefit of this qPCR assay is the ability to distinguish between the two hookworm species, $A$. duodenale and $N$. americanus. The hookworm species have long been grouped together in discussion since treatment is the same and it is very laborious to rear larvae to distinguish the two species. However, identifying the predominant species, $N$. americanus in this study, can contribute to better control measures as $N$. americanus transmission is frequent at defecation sites, because it cannot survive for long periods of time in the environment like A. duodenale [18]. The DNA concentration of $A$. duodenale in stool samples was higher than with $N$. americanus. This is consistent with the higher egg output per female $A$. duodenale worm per day [18]. $A$. duodenale has also been associated with more severe symptoms and greater blood loss with increased anemia [19]. This has important implications for anemia and long-term morbidity when higher $A$. duodenale DNA concentrations in asymptomatic individuals are discovered. A study of hookworm infection in Malawian preschool children used real-time PCR $\mathrm{C}_{\mathrm{t}}$ values as a proxy for infection load and found an association between increased load of $A$. duodenale and iron deficiency though the sample size was limited [20]. These differences and the lack of data on post-treatment efficacy stratified by hookworm species support the need for better characterization of prevalence by methods such as the qPCR assay [21].

Another benefit of qPCR is the ability to detect helminth DNA in stool without the presence of eggs. Since intestinal worms are continuously shedding and releasing DNA, amplification of DNA from stool is possible and increases parasite detection. However, most DNA 
from stool samples is overwhelmingly derived from the high density of eggs.

Results from this study also showed that qPCR is able to detect multiple parasite species at a higher rate than microscopy. The overwhelming majority of the study population tested positive for at least two different infections by qPCR. At most, microscopy detected a maximum of three species in the same sample, while qPCR was able to detect four. This has important population health implications for the implementation of mass drug administrations and for decisions related to choosing appropriate anti-parasitic drugs based on more accurate qPCR epidemiological data [22]. This study determined that G. lamblia has a high prevalence in this population, but might not be addressed with traditional community-based mass drug administrations that tend to target soil-transmitted helminths [22, 23]. Giardiasis commonly occurred with Ascariasis and hookworm species in dually infected subjects. The data on coinfection with $A$. lumbricoides or hookworms and $G$. lamblia is conflicting, with both positive and negative associations described in the literature [24, 25]. This could be attributed to the differences in water supply and hygiene in the different locations studied. Modulation of immune response in polyparasitism, in which infection with one parasite alters the immune response to another, has also been described and further supports the need for accurate diagnosis of polyparasitism in a population when planning interventions like a hookworm vaccine [25-27].

\section{Study limitations}

A limitation of the study was in the comparison of the hookworm egg count and the hookworm DNA concentration (Fig. 2). Since the ova of the two species are not distinct enough to be distinguishable by microscopy, it is difficult to estimate the egg output with co-infections also complicating the correlation. However, it is meant as a broad overview of the relationship of egg count and DNA concentration found in the stool samples. Another limitation of this study is the inability to determine whether $\mathrm{qPCR}$ is detecting residual DNA from previous infections and true prevalence in an endemic area is being overestimated. However, the constant peristalsis of the intestinal tract promotes evacuation of dead parasites and decreases the false positive rates detected by qPCR.

As in a previous Ecuador study, an internal control was not available [12]. This increases the possibility of missing positive samples due to inhibition or errors in extraction. The results of this pilot project did not appear to be impacted by PCR inhibition, given the general increase in detection by $\mathrm{qPCR}$, as seen in previous work [12].

\section{Conclusions}

As the use of molecular diagnostics in determining intestinal parasitic infections has become more common, multi-parallel qPCR assay offers a broad coverage of the most common intestinal parasites including the soiltransmitted helminths and pathogenic protozoa. Not including the cost of the instrument, this assay is less expensive than microscopy and requires less sophisticated supplies than multiplex assays so it can be effectively transferred to relatively resource-limited areas as previously demonstrated [12]. This high-throughput technique is designed to operate in a centralized location, such as a university or local hospital, and can be useful in targeting the community as a public health platform to actively survey current trends in parasitic infections and polyparasitism. Since $30 \%$ of the population worldwide are infected with intestinal parasites that can be associated with developmental delays, stunted growth, and decreased work productivity, there is ample opportunity for the use of this assay as a powerful tool in epidemiological studies [2-5].

This is the first time use of multi-parallel qPCR in Argentina and has demonstrated the high prevalence of intestinal parasites in a peri-urban area. Multi-parallel qPCR will be implemented to further investigate the relationships between different intestinal parasites that occupy the same anatomic niche and their impact on morbidity.

\section{Competing interests}

RM receives consulting fees from Lupin Pharmaceuticals, Inc. No funding for this project or paper was received by competing interests. The authors alone are responsible for the content and writing of the paper.

\section{Authors' contributions}

$\mathrm{ROC}, \mathrm{AK}$, and RM conceived and designed the experiments. RJ, ROC, PV, PC, $M J, P B$, and RM performed the experiments. ROC, RJ, and RM analysed the data. PC, MJ, JN and PV contributed reagents/materials/analysis tools. ROC, $R J, A K, A E$, and RM wrote the paper. All authors read and approved the final manuscript.

\section{Acknowledgements}

We are grateful to all the laboratory technicians of the IIET staff for their cooperation and help during the study period. We also thank the National School of Tropical Medicine, Baylor College of Medicine and Fundación Mundo Sano for their support.

\section{Author details}

${ }^{1}$ Instituto de Investigaciones en Enfermedades Tropicales - Universidad Nacional de Salta/CONICET, Oran, Argentina. ${ }^{2}$ Cátedra de Química Biológica, Facultad de Ciencias Naturales, Universidad Nacional de Salta, Oran, Argentina. ${ }^{3}$ National School of Tropical Medicine, Baylor College of Medicine, Houston, TX, USA. ${ }^{4}$ Fundación Mundo Sano, Buenos Aires, Argentina.

Received: 15 May 2015 Accepted: 7 July 2015

Published online: 17 July 2015

\section{References}

1. Preventive chemotherapy in human helminthiasis. http:// whqlibdoc.who.int/publications/2006/9241547103_eng.pdf. Accessed March 19, 2015. 
2. Brooker S, Clements ACA, Bundy DAP. Global epidemiology, ecology and control of soil-transmitted helminth infections. Adv Parasitol. 2006;62:221-61.

3. Hotez PJ, American Society for Microbiology. Forgotten people, forgotten diseases: the neglected tropical diseases and their impact on global health and development. 2nd ed. Washington, DC: ASM Press; 2013. xviii, 255 p. p.

4. Papier K, Williams GM, Luceres-Catubig R, Ahmed F, Olveda RM, McManus DP, et al. Childhood malnutrition and parasitic helminth interactions. Clin Infect Dis. 2014;59(2):234-43.

5. Ahmed A, Al-Mekhlafi HM, Al-Adhroey AH, Ithoi I, Abdulsalam AM, Surin J. The nutritional impacts of soil-transmitted helminths infections among Orang Asli schoolchildren in rural Malaysia. Parasit Vectors. 2012;5:119.

6. Smith $J$, Brooker S. Impact of hookworm infection and deworming on anaemia in non-pregnant populations: a systematic review. Trop Med Int Health. 2010;15(7):776-95.

7. Cimino RO, Krolewiecki A. The epidemiology of human strongyloidiasis. Curr Trop Med Rep. 2014;1(4):216-22. doi:10.1007/s40475-014-0035-6.

8. Krolewiecki AJ, Ramanathan R, Fink V, McAuliffe I, Cajal SP, Won K, et al. Improved diagnosis of Strongyloides stercoralis using recombinant antigen-based serologies in a community-wide study in northern Argentina. Clin Vaccine Immunol. 2010;17(10):1624-30.

9. Machicado JD, Marcos LA, Tello R, Canales M, Terashima A, Gotuzzo E. Diagnosis of soil-transmitted helminthiasis in an Amazonic community of Peru using multiple diagnostic techniques. Trans R Soc Trop Med Hyg. 2012;106(6):333-9.

10. Barda B, Cajal P, Villagran E, Cimino R, Juarez M, Krolewiecki A, et al. Mini-FLOTAC, Kato-Katz and McMaster: three methods, one goal; highlights from north Argentina. Parasit Vectors. 2014;7:271.

11. Barda B, lanniello D, Zepheryne $H$, Rinaldi L, Cringoli G, Burioni R, et al. Parasitic infections on the shore of Lake Victoria (East Africa) detected by Mini-FLOTAC and standard techniques. Acta Trop. 2014;137:140-6.

12. Mejia R, Vicuña Y, Broncano N, Sandoval C, Vaca M, Chico M, et al. A novel, multi-parallel, real-time polymerase chain reaction approach for eight gastrointestinal parasites provides improved diagnostic capabilities to resource-limited at-risk populations. Am J Trop Med Hyg. 2013;88(6):1041-7.

13. Verweij JJ, Brienen EAT, Ziem J, Yelifari L, Polderman AM, Van Lieshout L. Simultaneous detection and quantification of Ancylostoma duodenale, Necator americanus, and Oesophagostomum bifurcum in fecal samples using multiplex real-time PCR. Am J Trop Med Hyg. 2007;77(4):685-90.

14. Garcia L. Diagnostic medical parasitology. In: Diagnostic medical parasitology. 4th ed. Washington, D.C: ASM Press; 2001. p. 1092.

15. Hotez PJ. Holidays in the sun and the Caribbean's forgotten burden of neglected tropical diseases. PLoS Negl Trop Dis. 2008;2(5):e239.

16. De Silva NR, Brooker S, Hotez PJ, Montresor A, Engels D, Savioli L. Soil-transmitted helminth infections: updating the global picture. Trends Parasitol. 2003;19(12):547-51.

17. Socías ME, Fernández A, Gil JF, Krolewiecki AJ. Soil transmitted helminthiasis in Argentina. A systematic review. Medicina (B Aires). 2014;74(1):29-36.

18. Hoagland KE, Schad GA. Necator americanus and Ancylostoma duodenale: Life history parameters and epidemiological implications of two sympatric hookworms of humans. Exp Parasitol. 1978;44(1):36-49.

19. Albonico M, Stoltzfus RJ, Savioli L, Tielsch JM, Chwaya HM, Ercole E, et al. Epidemiological evidence for a differential effect of hookworm species, Ancylostoma duodenale or Necator americanus, on iron status of children. Int J Epidemiol. 1998;27(3):530-7.

20. Jonker FA, Calis JC, Phiri K, Brienen EA, Khoffi H, Brabin BJ, et al. Real-time PCR demonstrates Ancylostoma duodenale is a key factor in the etiology of severe anemia and iron deficiency in Malawian pre-school children. PLoS Negl Trop Dis. 2012;6(3):e1555.

21. Gulani A, Nagpal J, Osmond C, Sachdev HPS. Effect of administration of intestinal anthelmintic drugs on haemoglobin: systematic review of randomised controlled trials. BMJ. 2007;334(7603):1095.

22. Hotez P. Enlarging the "Audacious Goal": elimination of the world's high prevalence neglected tropical diseases. Vaccine. 2011;29 Suppl 4:D104-10.

23. Ferreira FS, Baptista-Fernandes T, Oliveira D, Rodrigues R, Neves E, Lima A, et al. Giardia duodenalis and soil-transmitted helminths infections in children in São Tomé and Príncipe: Do we think Giardia when addressing parasite control? J Trop Pediatr. 2015;61(2):106-12.

24. Blackwell AD, Martin M, Kaplan H, Gurven M. Antagonism between two intestinal parasites in humans: the importance of co-infection for infection risk and recovery dynamics. Proc R Soc B. 2013;280:20131671.
25. Hagel I, Cabrera M, Puccio F, Santaella C, Buvat E, Infante B, et al Co-infection with Ascaris lumbricoides modulates protective immune responses against Giardia duodenalis in school Venezuelan rural children. Acta Trop. 2011;117(3):189-95.

26. Geiger SM, Alexander ND, Fujiwara RT, Brooker S, Cundill B, Diemert DJ, et al. Necator americanus and helminth co-infections: further downmodulation of hookworm-specific type 1 immune responses. PLoS Negl Trop Dis. 2011;5(9):e1280.

27. Supali T, Verweij JJ, Wiria AE, Djuardi Y, Hamid F, Kaisar MM, et al. Polyparasitism and its impact on the immune system. Int J Parasitol. 2010;40:1171-6.

\section{Submit your next manuscript to BioMed Central and take full advantage of:}

- Convenient online submission

- Thorough peer review

- No space constraints or color figure charges

- Immediate publication on acceptance

- Inclusion in PubMed, CAS, Scopus and Google Scholar

- Research which is freely available for redistribution 